University of Massachusetts Amherst

ScholarWorks@UMass Amherst

1996

\title{
Cryofixing single cells and multicellular specimens enhances structure and immunocytochemistry for light microscopy
}

TI Baskin

DD Miller

JW Vos

JE Wilson

PK Hepler

Follow this and additional works at: https://scholarworks.umass.edu/biology_faculty_pubs

Part of the Biology Commons

\section{Recommended Citation}

Baskin, TI; Miller, DD; Vos, JW; Wilson, JE; and Hepler, PK, "Cryofixing single cells and multicellular specimens enhances structure and immunocytochemistry for light microscopy" (1996). Journal of Microscopy-Oxford. 30.

https://doi.org/10.1046/j.1365-2818.1996.135417.x

This Article is brought to you for free and open access by the Biology at ScholarWorks@UMass Amherst. It has been accepted for inclusion in Biology Department Faculty Publication Series by an authorized administrator of ScholarWorks@UMass Amherst. For more information, please contact scholarworks@library.umass.edu. 


\title{
Cryofixing single cells and multicellular specimens enhances structure and immunocytochemistry for light microscopy
}

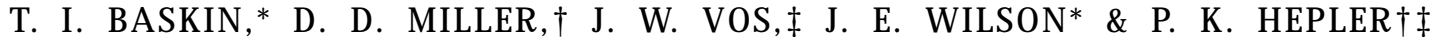 \\ *Division of Biological Sciences, University of M issouri, Columbia, M 0 65211, U.S.A. \\ †M olecular and Cellular Biology Program, and $¥ B$ Bology Department, U niversity of M assachusetts, \\ Amherst, MA 01003, U.S.A.
}

Key words. Actin, chemical fixation, cryofixation, immunocytochemistry,

methacrylate, microtubules, plunge freezing, pollen tubes, removable embedment,

roots, stamen hairs.

\section{Summary}

Cryofixation is widely held to be superior to chemical fixation for preserving cell structure; however, the use of cryofixation has been limited chiefly to electron microscopy. To see if cryofixation would improve sample structure or antigenicity as observed through the light microscope, we cryofixed Nicotiana alata and Lilium longiflorum pollen tubes and Tradescantia virginina stamen hairs by plunge freezing. After freeze-substitution, and embedding in butylmethylmethacrylate, we found using the light microscope that the superiority of cryofixation over chemical fixation was obvious. Cryofixation, unlike chemical fixation, did not distort cell morphology and preserved microtubule and actin arrays in a form closely resembling that of living cells.

A dditionally, to test further the usefulness of cryofixation for light microscopy, we studied the appearance of cells and the retention of antigenicity in a plunge-frozen multicellular organ. Roots of Arabidopsis thaliana were either chemically fixed or plunge frozen, and then embedded in the removable methacrylate resin used above. We found that plunge freezing preserved cell morphology far better than did chemical fixation, and likewise improved the appearance of both actin and microtubule arrays. Plunge-frozen roots also had cells with more life-like cytoplasm than those of chemically fixed roots, as assessed with toluidine-blue staining or high-resolution Nomarski optics. Damage from ice crystal formation could not be resolved through the light microscope, even in the interior of the root, 40-75 ${ }_{\mu} \mathrm{m}$ from

the surface We suggest that plunge freezing would enhance many investigations at the light microscope level, including those of multicellular organs, where damage from ice

Correspondence: Tobias I. Baskin, Tel. (1) 573882 0173; Fax (1) 5738820123. Present address of Deborah D. Miller: Department of Plant Cytology and Morphol$y$, Wageningen, The Netherlands. crystals may be less severe than artefacts from chemical fixation.

\section{Introduction}

It has long been realized that cryofixation is the method of choice for ultrastructural preservation of biological material. Most advances in cryo-techniques have been driven by the desire to come ever closer to perfect preservation. These advances include optimization of nearly every parameter in cryofixation and substitution, and technical developments such as cryo-ultramicrotomy and high-pressure freezing (Gilkey \& Staehelin, 1986; Menco, 1986; Hippe-Sanwald, 1993; Quintana, 1994). By contrast, cryofixed and freezesubstituted samples are rarely used in studies through the light microscope In particular, immunocytochemistry for the optical microscope, used in innumerable studies of cellular and subcellular localization, relies extensively on chemical fixation. Even though cryofixation and substitution have been shown with electron microscopy to eliminate a variety of artefacts caused by chemical fixation, the lack of widespread application of cryofixation to light microscopy implies that the differences between chemical and cryofixation might only be appreciable at the electron microscope level.

To compare the performance of cryofixation and chemical fixation for light microscopy, we have taken advantage of a resin system that has been developed to give excellent results with immunocytochemistry (Van de Kant et al., 1988; Gubler, 1989; Baskin et al., 1992). This resin system is based on a mixture of methyl- and butylmethacrylate, which can be removed readily after sectioning. For our comparison, we chose to examine germinating pollen tubes and Tradescantia stamen hair cells, because these single cell types have previously been shown to be preserved excellently for electron microscopy by plunge freezing (Lancelle et al., 
1986, 1987; Tiwari \& Polito, 1988; Lancelle\& Hepler, 1992). We find for light microscopy that cryofixation preserves the structure of pollen tubes and stamen hairs better than does chemical fixation, as judged by cell morphology and by the localization with immunocytochemistry of tubulin and actin.

As a further objective, we also sought to examine through the light microscope the appearance of a multicellular specimen prepared by plunge freezing. Plunge freezing is typically considered unsuitable for multicellular organs such as plant roots because acceptable preservation is confined to a thin surface layer of $5-10{ }_{\mu} \mathrm{m}$. Deeper than this, regardless of the specific methods used for freezing or substitution, damage from ice crystals cannot be avoided (Gilkey \& Staehelin, 1986; Sitte et al., 1987; Kaeser et al., 1989). Instead of plunge freezing, it is recognized that to freeze multicellular structures optimally one must use techniques such as high-pressure or propane jet freezing, which can prevent damaging ice crystal formation for considerable depths within the specimen (Gilkey \& Staehelin, 1986; Ding et al., 1991). However, these techniques are not perfect, requiring cryoprotectants, expen sive apparatus, and causing artefacts of their own (for discussion, see Dahl \& Staehelin, 1989; Hyde et al., 1991). By contrast, plunge freezing is well characterized, can be done with relatively simple, homemade apparatus, and minimizes disruption to the sample prior to freezing (Lancelle et al., 1986; Howard \& O'Donnell, 1987; Sitte et al., 1987; Ridge, 1990; Ryan, 1992).

While damage from ice crystals can be overwhelming at the high magnification sof electron microscopy, the size of ice crystals in cryofixed tissue that has been optimally frozen is typically $100 \mathrm{~nm}$ or less (Ryan, 1992), which is smaller than the limit of resolution for optical microscopy. Nor does crystal size inevitably increase with depth; for example, in plungefrozen samples that were $500 \mu \mathrm{m}$ thick, mean ice crystal diameter was essentially constant throughout the sample (Ryan, 1992). Moreover, chemical fixation, while not causing ice crystal damage, often lessens antigenicity and may cause many artefacts, including vesiculation of membranes (Mersey \& McCully, 1978; Hyde et al., 1991), deformation of structures (Skaer \& Whytock, 1976; He \& Wetzstein, 1995) and redistribution of proteins (Shopfer \& Hepler, 1991; Melan \& Sluder, 1992), any of which degrade the image as compared with that from live cells. Therefore, we wished to see whether ice crystals resulting from plunge freezing a multicellular organ caused damage that was, at the light microscope level, less severe than the alterations caused by chemical fixation.

We chose the roots of Arabidopsis thaliana (hereafter referred to by its vernacular name, arabidopsis), because they are thin (c. $140 \mu \mathrm{m}$ ) and because we have extensive experience with this material prepared by chemical fixation (Baskin et al., 1992, 1994). We find that the appearance of cryofixed roots is equal or superior to that of chemically fixed roots, as judged by the straightness of cell walls and the preservation of cytoskeletal arrays, and in well-frozen roots we could not optically resolve ice crystal damage in any region of the sample. We suggest that cryofixation of multicellular organs can usefully complement chemical fixation for studies through the light microscope

\section{Materials and methods}

\section{Plant material and growth}

Fresh pollen from greenhouse-grown Lilium longiflorum and Nicotiana alata was germinated for $2-3 \mathrm{~h}$ prior to cryofixation. Tradescantia virginiana plants were grown at $18-22^{\circ} \mathrm{C}$ under an 18 -h day in a growth chamber. Stamen hairs were dissected from anthers of young flower buds. Arabidopsis thaliana L. (Heynh) ecotype Columbia was grown vertically on nutrient solidified agar in Petri dishes for 6-10 days, as described by Baskin \& Bivens (1995).

\section{Cryofixation and freeze-substitution}

Samples were cryofixed by plunging into liquid propane held at $-180^{\circ} \mathrm{C}$. For pollen tubes and stamen hairs, the apparatus described by Lancelle et al. (1986) was used. For arabidopsis roots, a similar apparatus was constructed, with the major difference being that gravity was used for plunging, not a rotary solenoid. The gravity-based plunger was calculated to move the sample at $2 \mathrm{~m} \mathrm{~s}^{-1}$ at entry into the propane. For substitution, pollen tubes and stamen hairs were substituted at $-80^{\circ} \mathrm{C}$ in a custom-built apparatus using liquid nitrogen for cooling. Roots were substituted at $-76^{\circ} \mathrm{C}$ in a chest-type freezer.

All samples were supported on 32 gauge, nichrome wire loops, coated with Formvar as described in Lancelle et al. (1986). Recently, we have found that compared with Formvar, coating loops with agar $(2.5 \%$ in water; Tiwari \& Polito, 1988) increases the yield of well-frozen pollen tubes. Pollen tubes were harvested and frozen as described by Lancelle et al. (1986). Stamen hairs were dissected in a standard culture medium ( $5 \mathrm{~mm}$ Hepes, $1 \mathrm{~mm} \mathrm{KCl}, 1 \mathrm{~mm}$ $\mathrm{MgCl}_{2}$ and $0.1 \mathrm{~mm} \mathrm{CaCl}$ ) and put on Formvar loops in a droplet of this medium plus $0.025 \%$ Triton-X100. Inclusion of Triton helped to wet the cuticle so that hairs would sink through the droplet and adhere to the Formvar. To increase retention of stamen hairs, the Formvar was coated with $2 \mathrm{mg} \mathrm{mL}^{-1}$ poly-L-lysine. Most of the culture medium was carefully wicked away immediately prior to plunging. After cryofixation, pollen and stamen hairs were substituted in media with a molecular sieve for $36 \mathrm{~h}$, and then brought up to room temperature slowly over $18 \mathrm{~h}$. In some experiments, stamen hairs were warmed only to $-20^{\circ} \mathrm{C}$ and infiltrated at this temperature For pollen and stamen hairs, pure acetone was used as the substitution medium; in addition, for stamen hairs, acetone containing either $4 \%$ paraformaldehyde (PFA) 
or $0.1 \%$ glutaral dehyde (GA) was used. Fixatives were diluted from $37 \%$ PFA or $25 \%$ GA (aqueous, EM-grade stocks).

Arabidopsis roots were frozen by laying the apical few millimetres of the root on the loop's Formvar surface, and cutting the root outside the loop with a sharp razor. We found that films for coating wire loops could be cast successfully from a $0.25 \%$ Formvar solution, presumably thinner than those cast from the $1.2 \%$ solution described by Lancelle et al. (1986). Roots were typically substituted for 48 $\mathrm{h}$ in freshly opened acetone (molecular sieve was not used) and allowed to warm up to $-4{ }^{\circ} \mathrm{C}$ over $18 \mathrm{~h}$, and then to room temperature over 1-2 h. Other substitution media were also used, including ethanol, methanol and tetrahydrofuran. In some trials, solvents contained $1 \%$ acidified dimethoxypropane (DMP) to remove water (Kaeser, 1989). In other trials 4\% PFA was added to ethanol by dissolving a pellet of $\mathrm{NaOH}$ in ethanol, followed by addition of granular PFA.

\section{Infiltration and embedding}

All steps after substitution were usually done at room temperature. Samples were rinsed in fresh substitution medium and infiltrated with a graded resin/substitution medium mixture (25\%, 50\%, 75\%), with 15-85 min (hairs) or $1 \mathrm{~h}$ (pollen, roots) per step, and three changes of $100 \%$ resin, with 15-135 min (hairs) or several hours (pollen, roots) per step, except that the second or third step went overnight. Resin comprised $80 \%$ butylmethacrylate, 20\% methylmethacrylate (Aldrich Chemical Corp., Milwaukee, WI), $0.5 \%$ benzoinethylether (Aldrich) and $10 \mathrm{~mm}$ dithiothreitol (DTT) (Baskin et al., 1992). In some trials, the DTT was omitted. The resin mixture was degassed with nitrogen for $20 \mathrm{~min}$ and stored at $-20^{\circ} \mathrm{C}$.

For embedment, the stem of the wire loop was removed to place samples individually in BEEM ${ }^{\circledR}$ capsules. For flat embedments, in some experiments, the capsule was inverted, with the cap sealed with Parafilm. Later, similar capsules manufactured with flat bottoms were obtained (TAAB Laboratories Ltd., Aldermaston, Berks.). Resin was polymerized under long-wavelength UV light at $4{ }^{\circ} \mathrm{C}$. For pollen and stamen hairs, capsules were placed on a glass sheet about 4 $\mathrm{cm}$ above an 8-W source for $6 \mathrm{~h}$, whereas for roots, capsules were placed $5-10 \mathrm{~cm}$ beneath a $15-\mathrm{W}$ source for $4 \mathrm{~h}$.

\section{Chemical fixation}

Pollen tubes were grown as described above and fixed at room temperature for $1 \mathrm{~h}$ in 4\% PFA, $50 \mathrm{~mm}$ PIPES ( $\mathrm{pH} 7 \cdot 0$ ), $5 \mathrm{~mm}^{2} \mathrm{EGTA}$, $1 \mathrm{~mm} \mathrm{MgSO}_{4}, 40 \mathrm{~g} \mathrm{~mL}$ leupeptin, 1 mм phenyl- $\mu$ methylsulfonyl fluoride, $0 \cdot 25 \%$ casein and $10 \%$ sucrose. They were then dehydrated through a graded ethanol series with
30 min per step and overnight in 100\% ethanol. Dehydrated pollen tubes were infiltrated through a graded series $(25 \%$, $50 \%, 75 \%, 100 \%)$ of methacrylate in ethanol with $1 \mathrm{~h}$ per step, a 2-h $100 \%$ methacrylate step, followed by a final $100 \%$ step overnight. Pollen tubes were then placed in capsules with fresh methacrylate for polymerization as described above.

Stamen hairs were prepared on Formvar-coated loops as above and the loops were placed into one of the following fixatives: $4 \%$ PFA, $0.1 \% \mathrm{GA}$, or both, in $50 \mathrm{~mm}$ PIPES $(\mathrm{pH}$ 7.0), $5 \mathrm{~mm}$ EGTA and $1 \mathrm{~mm} \mathrm{MgSO}_{4}$. Fixation was at room temperature for $1-1 \cdot 75 \mathrm{~h}$. Stamen hairs were dehydrated through a graded ethanol series with 40 min per step, 100\% ethanol overnight, a 40 -min step in fresh $100 \%$ ethanol, and were then infiltrated in resin and polymerized as for the cryofixed stamen hairs.

For roots, seedlings were fixed for $2 \mathrm{~h}$ at room temperature in $4 \%$ PFA, $50 \mathrm{~mm}$ PIPES (pH 7.0) and $1 \mathrm{~mm} \mathrm{CaCl}_{2}$. The fixative solution was poured directly on the plates. After three 10-min rinses in the above solution minus the fixative, c. 3-mm apical root segments were encased in $2 \%(\mathrm{w} / \mathrm{v}$ in water) low melting agarose (Type VII, Sigma Chemical Co., St Louis, MO) and dehydrated in a graded ethanol series, with 15-30 min per step. In contrast to the original method (Baskin et al., 1992), the ethanol series did not contain DTT, because we have found no difference in the samples dehydrated with or without $1 \mathrm{~mm}$ DTT (J.E.W. and T.I.B., unpublished). Samples were infiltrated with 25\%, 50\%, 75\% resin in ethanol, with 30 min per step. Subsequent steps were done exactly as for freeze-substituted samples, described above, except that from $50 \%$ ethanol through to embedment, samples were kept at $-20^{\circ} \mathrm{C}$.

\section{Sectioning, staining and microscopy}

The embedded wire was carefully excised from around the sample. Because the brittleness of the plastic made this tedious, in some arabidopsis trials, we made loops with 36 gauge copper wire. Although copper reacts with LR White (S. Lancelle, personal communication, 1995), it does not react visibly with methacrylate. The thinner wire was readily cut within the blocks with a double-edged razor blade, resulting in faster sample trimming. Sections were cut dry on an ultramicrotome, placed in droplets of water and affixed to slides coated with 3-aminopropyltriethoxy silane (Angerer \& Angerer, 1991) by heating briefly on a slide warmer for 2$5 \mathrm{~min}$ at $60^{\circ} \mathrm{C}$. For pollen tubes, sections were $2-4 \mu \mathrm{m}$ thick, for stamen hairs $2-3 \mu \mathrm{m}$ and for roots $1.75 \mu \mathrm{m}$.

Some root sections were stained in $0.2 \%$ toluidine blue in borax and mounted in water. For immunocytochemistry, sections were extracted in acetone (22 min for pollen, 20$30 \mathrm{~min}$ for hairs, $10 \mathrm{~min}$ for roots) and then immediately rehydrated in phosphate-buffered saline (PBS) containing 0.05\% Tween-20 (PBS-Tween). A graded rehydration series was not used. For stamen hairs exposed to glutaraldehyde, 

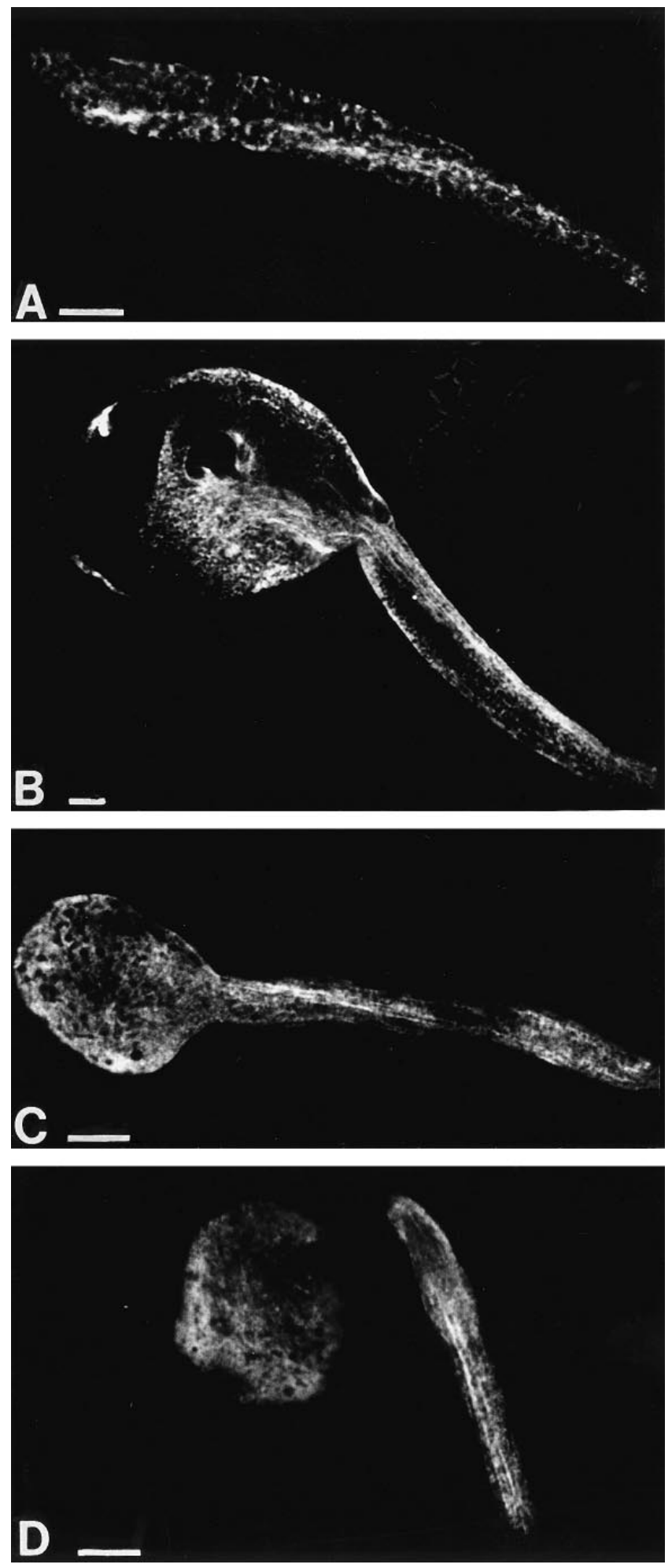

Fig. 1. Comparison of actin localization in chemically fixed vs. cryofixed pollen tubes embedded in butylmethylmethacrylate. Actin was localized with an antibody following removal of the embedment. (A) Chemical fixation of lily. Note that longitudinal actin filaments are coarse and clumped. (B-D) Cryofixation in (B) lily, (C,D) tobacco; either in chemical fixation or in freeze-substitution, sections were treated with $\mathrm{NaBH}_{4}$ solution, $0 \cdot 1 \%$ (v/w) in PBS, for $30 \mathrm{~min}$. Sections were blocked (1\% BSA in PBS-Tween for pollen and stamen hairs, $0.1 \%$ Tween-20 in PBS for roots), incubated in primary antibody $\left(2 \mathrm{~h}\right.$ at $\left.37^{\circ} \mathrm{C}\right)$, rinsed in PBSTween $(3 \times 10 \mathrm{~min})$, incubated in secondary antibody $(2 \mathrm{~h}$ at $\left.37^{\circ} \mathrm{C}\right)$, rinsed in PBS-Tween $(3 \times 10 \mathrm{~min})$ and mounted in a commercial antifading reagent (Vectashield; Vector laboratories, Burlingame, CA).

Antibodies used were as follows. Monoclonal anti- $\alpha$ tubulin, raised against chicken brain microtubules (DM1 A, Sigma) and used at 1:100 for pollen and stamen hairs, or raised against sea-urchin axonemes (B-5-1-2, Sigma) and used 1:1000 for roots; monoclonal anti-actin, raised against pea actin (Andersland et al., 1994), the gift of Dr R. J. Cyr (Pennsylvania State University), and used throughout at 1:10; and as a secondary, goat anti-mouse Fab fragments conjugated with Cy3 (Jackson Immuno-Research Laboratories, West Grove, PA), used at 1:200.

Fluorescence microscopy of pollen tubes and stamen hairs (except where noted) was done on a confocal laser scanning microscope (Bio-Rad MRC 600), and for roots was done with conventional epifluorescence (Zeiss Axioplan). For both microscopes, fluorescence from Сy 3 was observed using the standard rhodamine filter cube. The Nomarski images of roots were obtained with a $100 \times 1 \cdot 3-N A$ objective, and a 1.4-NA condenser oiled to the slide and stopped down by about $10 \%$.

\section{Results}

\section{Pollen tubes}

Pollen tubes are typically observed at the light microscope level in whole mount preparations, with the cell wall extracted to provide the needed access for the antibodies (e.g. Tiwari \& Polito, 1988; He \& Wetzstein, 1995). Because cell wall extraction apparently has deleterious effects on cell structure, we examined pollen tubes that were processed for immunofluorescence in sections, rather than in whole mounts. At the level of structural preservation, cryofixation clearly surpassed chemical fixation. In chemically fixed material, the tube cell wall was often wavy, with the plasma membrane frequently detached in places from the wall, and, as usual with chemical fixation, the clear zone at the tip was lost; however, with cryofixation, the edges of the tube cell wall were smooth, with few areas of membrane detachment,

note the fine actin filaments running longitudinally from the grain into the tube and throughout the length of the tube. Single confocal images except (B), which is a summed Z-series of seven images, with $0.5 \mu \mathrm{m}$ per image. Scale bars $=10 \mu \mathrm{m} ;(A, C, D)$ $810 \times$, (B) $435 \times$. 
and the clear zone at the tip was reliably retained (not shown). Similarly, cryofixation also surpassed chemical fixation for the localization of actin, as performed with an anti-actin antibody (Andersland et al., 1994). In chemically fixed pollen tubes, actin filaments were detected, but the filamentous staining appeared coarse and fragmented
(Fig. 1A). By contrast, for cryofixed pollen, fine actin filaments were localized in the grain and throughout the pollen tube (Fig. 1B-D). Cryofixation led to a similar pattern in both lily (Fig. 1B) and tobacco (Fig. 1C,D). In these images, the nonfilamentous staining probably results from affinity of the antibody for G-actin, which is known to be abundant in
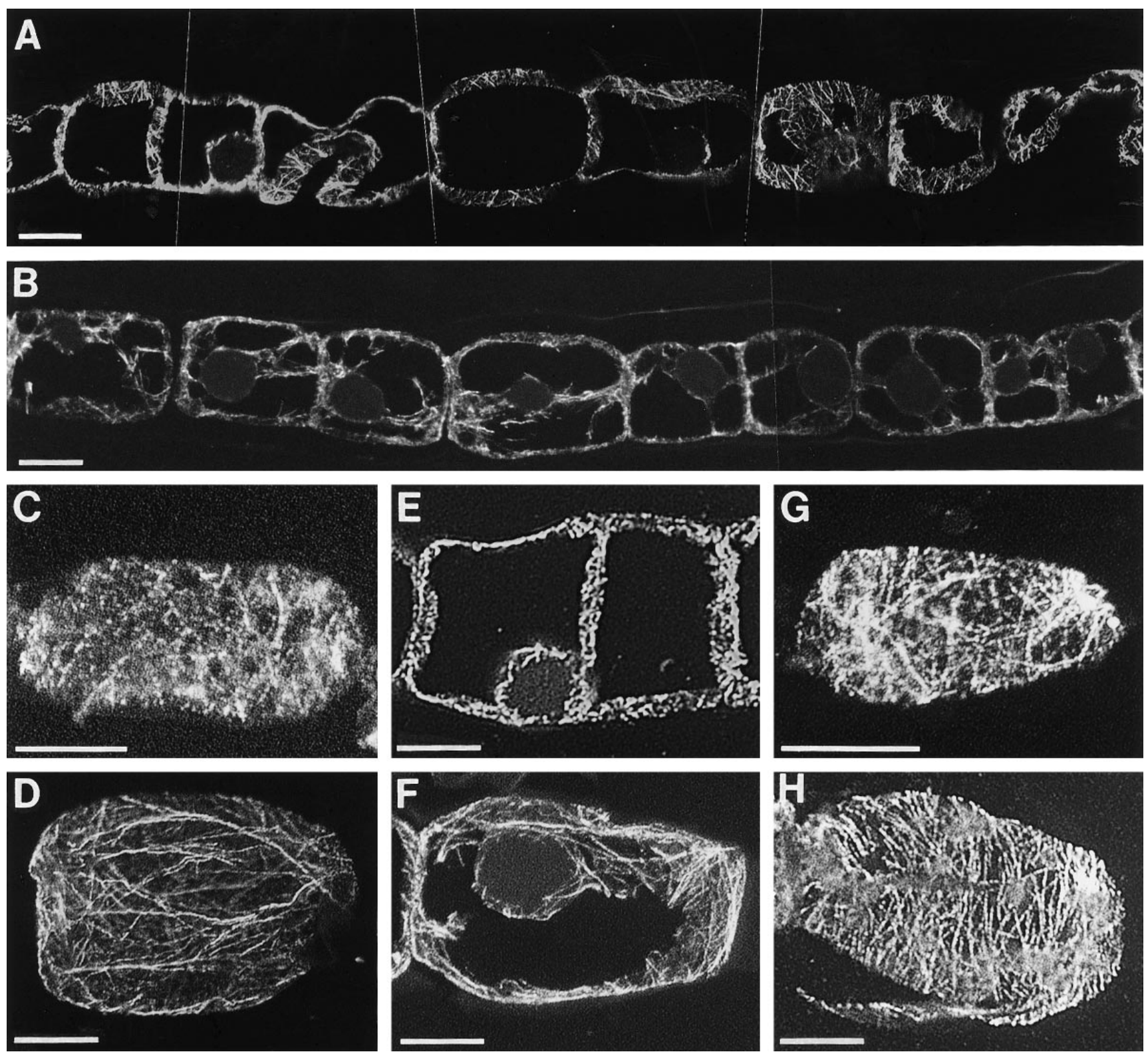

Fig. 2. Comparison of actin and tubulin localizations in chemically fixed vs. cryofixed stamen hairs embedded in butylmethylmethacrylate. Actin and tubulin were localized with antibodies following removal of the embedment. Panels in pairs, with upper panels $(A, C, E, G)$ showing chemical fixation and lower panels $(B, D, F, H)$ showing cryofixation. (A - F) Sections stained with anti-actin: (A,B) Morphological overview; (C-D) cortical regions; $(E-F)$ medial regions. Note disruption of cell shape and cytoplasmic strands with chemical fixation, and the appearance of abundant fine actin filaments with cryofixation. (G-H) Sections stained with anti-tubulin. Note abundant and well-preserved cortical microtubules only in the cryofixed preparations. Chemical fixation in (A,C,D) 4\% PFA, or (G) 4\% PFA plus $0.1 \%$ GA; freeze substitution in acetone (B) only, (D,F) plus $4 \%$ PFA, or (H) plus 0.1\% GA. Confocal images (except for B, taken with conventional epifluorescence, Nikon Diaphot), with summed Z-series used for $C$ (seven images) and F (eight images), with $0.5 \mu \mathrm{m}$ per image. Scale bars $=10 \mu \mathrm{m}(\mathrm{A}, \mathrm{B}) 940 \times,(\mathrm{C}) 1670 \times,(\mathrm{D}, \mathrm{E}, \mathrm{F}, \mathrm{H})$ $1240 \times,(G) 2080 x$ 
pollen (Andersland et al., 1994), and the frequent rounded, dark interruptions of the stain reflect the presence of abundant plastids and other organelles. The fine actin staining seen with cryofixation resembles what has recently been seen when filamentous actin is stained, by microinjection of fluorescent phalloidin, in living pollen tubes (Miller et al., unpublished observations). In agreement with our results for pollen tubes, Czymmek et al. (1996) have found for fungal hyphae that cryofixation improves structural preservation and antigenicity, as assessed by light microscopy of sectioned material.

\section{Stamen hairs}

Chemical fixation of stamen hairs did not preserve their morphology well. In particular, the plasma membrane was sometimes dissociated from the cell walls (not shown), cell shapes were often deformed and only few cytoplasmic strands remained (Fig.2A). By contrast, with cryofixation, separations of the membrane from the wall were rare, cell shapes looked normal and many strands remained (Fig. 2B). In general, improvements in morphology and antigenicity seen with cryofixation occurred regardless of whether a fixative was included at substitution, or whether infiltration was performed at $-20^{\circ} \mathrm{C}$.

Chemical fixation did permit localization of actin. However, similarly to pollen tubes, coarse and fragmented bundles predominated, both in the cortical cytoplasm (Fig.2C) and around the nucleus (Fig. 2E). Fine arrays of actin filaments were rarely seen. By contrast, with cryofixation, fine actin filaments were routinely visible. These filaments permeated the cytoplasm, appearing random in the cell cortex (Fig. 2D) and in the perinuclear region (Fig. $2 \mathrm{~F}$ ), but running parallel to the axis of cytoplasmic strands (Fig. 2B,F). Furthermore, fine actin filaments were resolved in the mitotic spindle, parallel to the microtubules (not shown). Chemical fixation also did not give optimal tubulin labelling: although spindles and phragmoplasts of dividing cells were well stained (not shown), cortical microtubules were usually absent or sparse, and those remaining were fragmented or bundled (Fig. 2G). By contrast, in cryofixed cells, cortical microtubules were labelled abundantly and routinely (Fig. $2 \mathrm{H}$ ). For both actin and tubulin, the images from cryofixation, as opposed to chemical fixation, closely resemble the appearance of these cytoskeletal proteins in living stamen hair cells (Hepler et al., 1993).

\section{A rabidopsis roots}

To assess the appearance through the light microscope of a cryofixed, freeze-substituted multicellular organ, we chose the primary root of $A$. thaliana. Although arabidopsis roots have been found generally to be well preserved for immunocytochemistry with chemical fixation and the methacrylate resin used here (Baskin et al., 1992, 1994), we were originally driven to investigate cryofixation because of persistent problems. For example, with chemical fixation, the cell walls of large cells were usually extensively wrinkled, thus distorting the cortical microtubule arrays (Fig. 3A). Furthermore, there was a sharp drop in staining intensity at the transition between zones of cell division and rapid elongation, especially in epidermis and cortex (Fig.3C). Staining intensity usually recovered by some point in the rapid elongation or mature zone.

In cryofixed roots, the cell walls of large cells were usually straight, and cortical microtubules were brightly stained and their organization clear (Fig. 3B,D,E); nor was there any large loss of staining intensity at the transition between zones of division and rapid elongation (Fig.3D). Straight walls occurred with all of the substitution media tested (tetrahydrofuran, methanol, ethanol or acetone). The example showing loss of stain intensity (Fig. 3C) comes from a chemically fixed root that, in addition, was then cryofixed and freeze-substituted to avoid dehydration. The loss of staining in such roots was indistinguishable from that of chemically fixed roots processed as usual, which means that the loss of staining resulted from inadequate fixation, not through dehydration. Additionally for this treatment, the walls of large cells were wrinkled more than with cryofixation alone, but less than with chemical fixation alone, which implies that wrinkling is caused by both chemical fixation and dehydration.

Although reasonable results were obtained with methanol and ethanol, roots substituted in acetone appeared superior in having straighter cell walls and fewer regions of apparent cellular disruption (not shown). Nevertheless, a problem with acetone substitution was that roots tended to split along cell walls during sectioning (Fig. 3D). This wall splitting was often so severe that much of the root was lost during sectioning. However, as a result of wall splitting, isolated cell fragments were present on the slide, occasionally providing matchless views of cortical microtubule arrays (Fig. 3E). In some roots, cells in innermost layers appeared to be damaged, based on aberrant shapes and microtubule patterns (not shown); nevertheless, in many roots, no evidence was seen of cellular damage, even in interior tissues, 40-75 $\mu \mathrm{m}$ from the surface. In the apical region of the root where microtubule arrays are well localized with conventional fixation (Fig.4A), arrays in cryofixed roots appear identically well preserved through the full depth of the organ (Fig. 4B).

To assess further the usefulness of cryofixed roots, we examined the distribution of actin. In chemically fixed roots, localization of actin with the antibody showed cells in the stele to have bright longitudinal actin bundles and diffuse fluorescence, while cortical and epidermal cells had scant staining of any kind (Fig. 4C). By contrast, in cryofixed roots, all cell types showed a fine filamentous network, permeating 

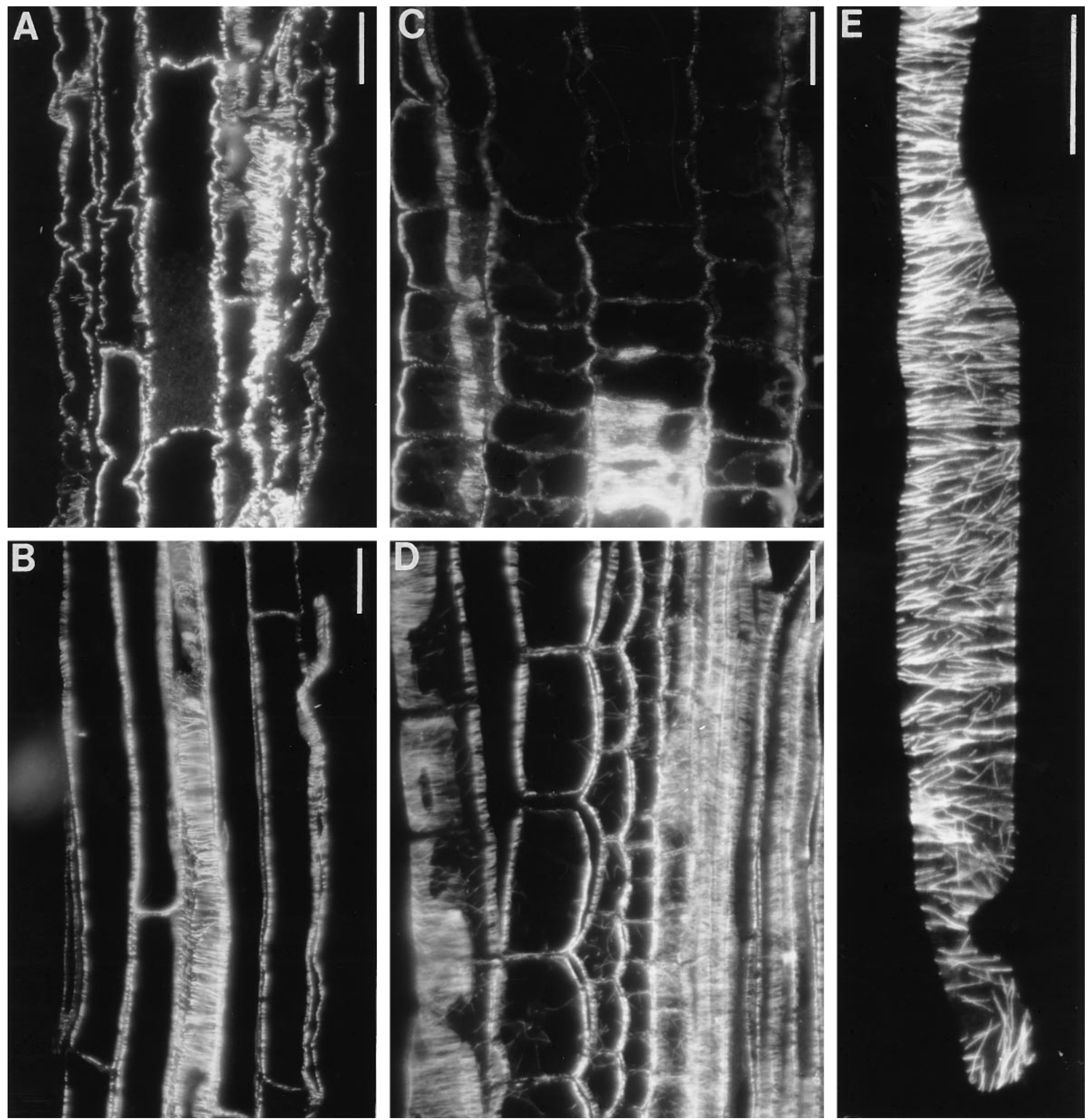

Fig. 3. Comparison of chemical fixation vs. plunge freezing on the localization of microtubules in the roots of $A$. thaliana. Tubulin was localized with antibodies following removal of the embedment. Cryofixed roots were freeze-substituted in DMP-acetone. Cortical microtubule arrays: $(A, C)$ Chemical fixation, $(B, D, E)$ cryofixation. $(A, B)$ Region of rapid elongation; note the wrinkling of cell walls with chemical but not cryofixa-tion. (C,D) Transitional region between zones of cell division and rapid elongation; note the loss of staining intensity as the zone of rapid elonga-

tion begins for chemical but not cryofixation. Root for panel (C) was cryofixed following chemical fixation. (E) Cell fragment separated from the tissue in a cryofixed root; note the presence of divergent microtubules among the predominantly transverse array. Scale bars $=20 \mathrm{~m}$, (A,B)

560 , (C,D) 575 , (E) 1120 . 

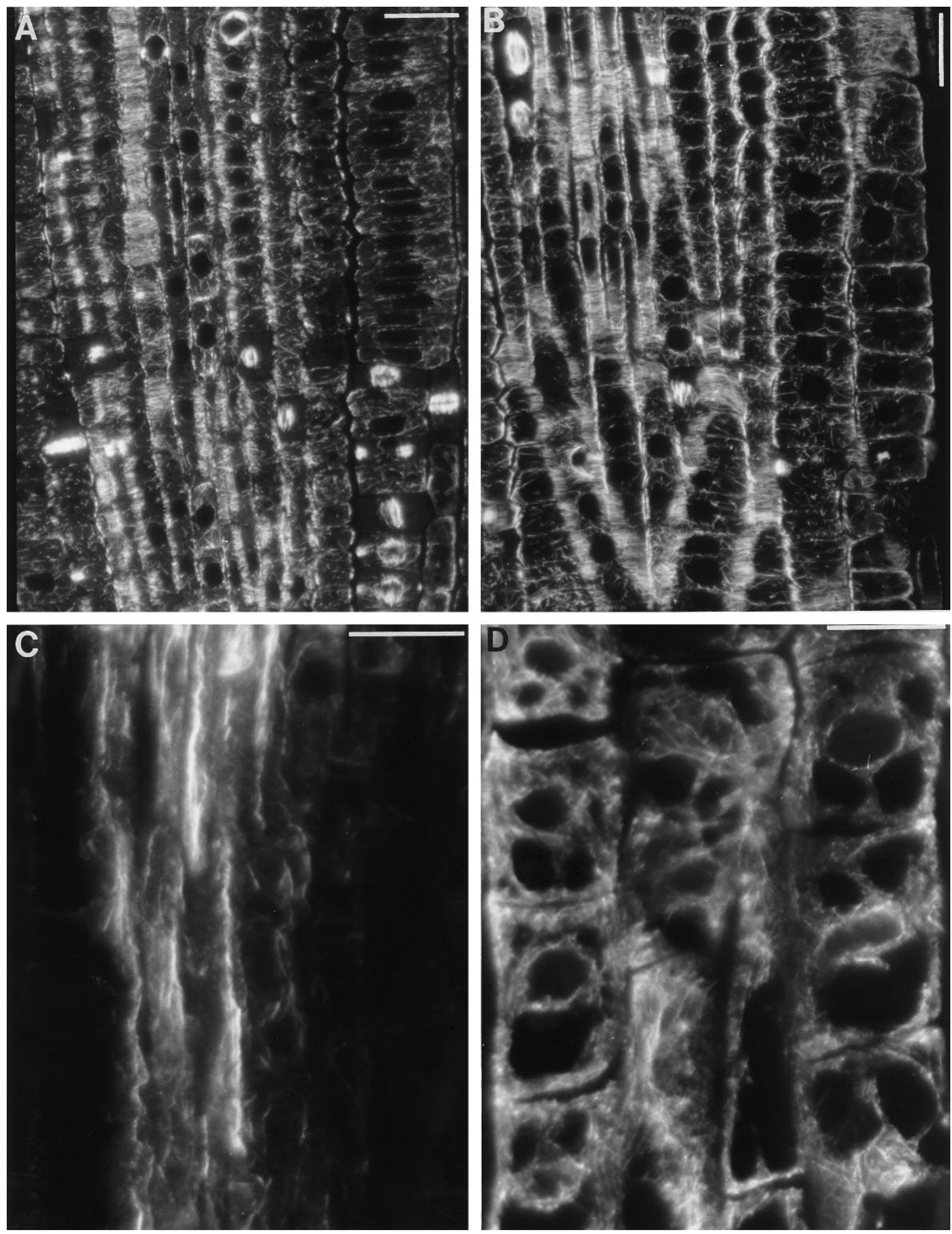
the cytoplasm and surrounding the nucleus (Fig.4D), reminiscent of that observed in living plant cells (Hepler et al., 1993). Stelar cells in cryofixed tissue had, in addition to fine filamentous staining, longitudinal actin bundles (not shown), but these bundles were less dominant than those seen in chemically fixed cells (Fig. 4C).

To compare morphology between chemically fixed and plunge-frozen roots, we examined the overall appearance of the cytoplasm. With toluidine-blue staining, the cytoplasm in chemically fixed roots appeared grainy and ragged, whereas that of cryofixed roots appeared smooth (Fig. 5A,B). Similarly, viewed with Nomarski optics at high NA, chemically fixed cytoplasm appeared grainy, whereas cryofixed cytoplasm appeared smooth (Fig.5C-F). In cryofixed but not chemically fixed roots, large cells had fine transvacuolar strands, appearing almost as though they remained under tension (Fig.5C,D). Further, with chemical fixation, dividing cells, which have very dense cytoplasm, appeared nearly featureless and had smooth spherical nuclei; but with cryofixation, such cells contained inclusions of various sizes and shapes, as expected for organelles, and had lumpy nuclei (Fig. 5E,F). Complex membrane systems and nonspherical nuclei are features known in certain living plant cell types to be lost with chemical fixation (Mersey \& McCully, 1978; McCully \& Canny, 1985). Additionally, in well-frozen roots, examination of cells with Nomarski optics at high NA failed to resolve holes from ice crystals (Fig. 5D,F).

\section{Discussion}

We have found that cryofixation by plunge freezing and freeze-substitution enhances the preservation of samples for light microscopical analysis. Our results with pollen tubes and stamen hairs are straightforward. These cell types are small enough to be reliably cryofixed by plunge freezing (Lancelle et al., 1986; Tiwari \& Polito, 1988; Lancelle \& Hepler, 1992). Moreover, cryofixation compared to chemical fixation has been repeatedly found to enhance the preservation of ultrastructure and to improve results with immunoelectron microscopy (Kiss \& McDonald, 1993; Nicolas \& Bassot, 1993). Therefore, it is not surprising that cryofixation of single cells surpasses chemical fixation for light microscopy. Similarly, comparisons of chemically fixed vs. plungefrozen fungal hyphae (Raudaskoski et al., 1991; Roberson \& Vargas, 1994), including a study where substitution was followed by embedment in the removable resin system used here (Czymmek et al., 1996), found cryofixed material was far superior to chemical fixation for immunocytochemistry at the light microscope level.

As for single cells, we have also found for a multicellular specimen, the arabidopsis root, that plunge freezing is a valuable alternative to high-pressure freezing or chemical fixation for light microscopy. Plunge freezing for multicellular specimensis now generally dismissed, owing to the inevitable occurrence of ice crystal damage below a thin surface layer (Kiss \& McDonald, 1993; Nicolas \& Bassot, 1993). This dismissal arises from examination of ultrastructure at the high magnifications of electron microscopy, where the disruptions from ice crystal formation manifestly impair observation. However, plunge freezing multicellular objects need not likewise be dismissed for light microscopy because the distortions from unresolved ice crystal holes may be preferable to the lost antigenicity or structural artefacts caused by chemical fixation. Indeed, the historical development of cryofixation, spurred by histologists seeking to surpass chemical fixation, was conducted exclusively through light microscopy. Early investigations of cryofixation where multicellular plant and animal organs were plunge frozen, freeze-substituted and examined with light microscopy, reported no resolvable ice crystal damage in wellfrozen samples, and found morphological preservation that surpassed that obtained with chemical fixation (e.g. Feder $\&$ Sidman, 1958), although fixation with aldehydes was not used. These studies support the notion that plunge freezing is valid and valuable for light microscopical studies of multicellular organs.

For our work, we used a plunging system that has been shown to give excellent results at the electron microscope level with single cells (Lancelle et al., 1968), and that avoids several pitfalls, such as precooling the sample through cold air before entering the cryogen (Ryan, 1992). Nevertheless, the actual extent of ice crystal damage must be ascertained for each type of specimen. Even studies at the light microscope level are unlikely to tolerate massive ice crystal damage from poorly frozen samples. To check the quality of cryofixation of the roots, we cut ultrathin sections from the same blocks used for light microscopy and examined them in the electron microscope Although lack of osmication rendered the contrast low, the cytoplasm appeared honeycombed, as typically reported for ice crystal damage. We quantified the size of individual holes by measuring a distance across the hole that was intermediate between the longest and shortest dimension. We found for the holes in the nuclei and adjacent cytoplasm of 33 cells, spread over the

Fig. 4. Comparison of chemical fixation vs. plunge freezing on the localization of microtubules and actin in the roots of $A$. thaliana. Actin and tubulin were localized with antibodies following removal of the embedment. Cryofixed roots were freeze-substituted in DMP-acetone. (A,B) Microtubule localizations in (A) chemically fixed and (B) plunge-frozen roots; note the similarity of the appearance of the arrays, even in the interior of the tissue. (C,D) Actin localizations in (C) chemically fixed and (D) plunge-frozen roots; note with chemical fixation predominant bundles of actin in the narrow cells of the stele and no staining in epidermis or cortex, and in cryofixed roots, filamentous stain ing throughout the cytoplasm. Scale bars $=15 \mu \mathrm{m},(\mathrm{A}, \mathrm{B}) 875 \times,(\mathrm{C}, \mathrm{D}) 1405 \times$. 

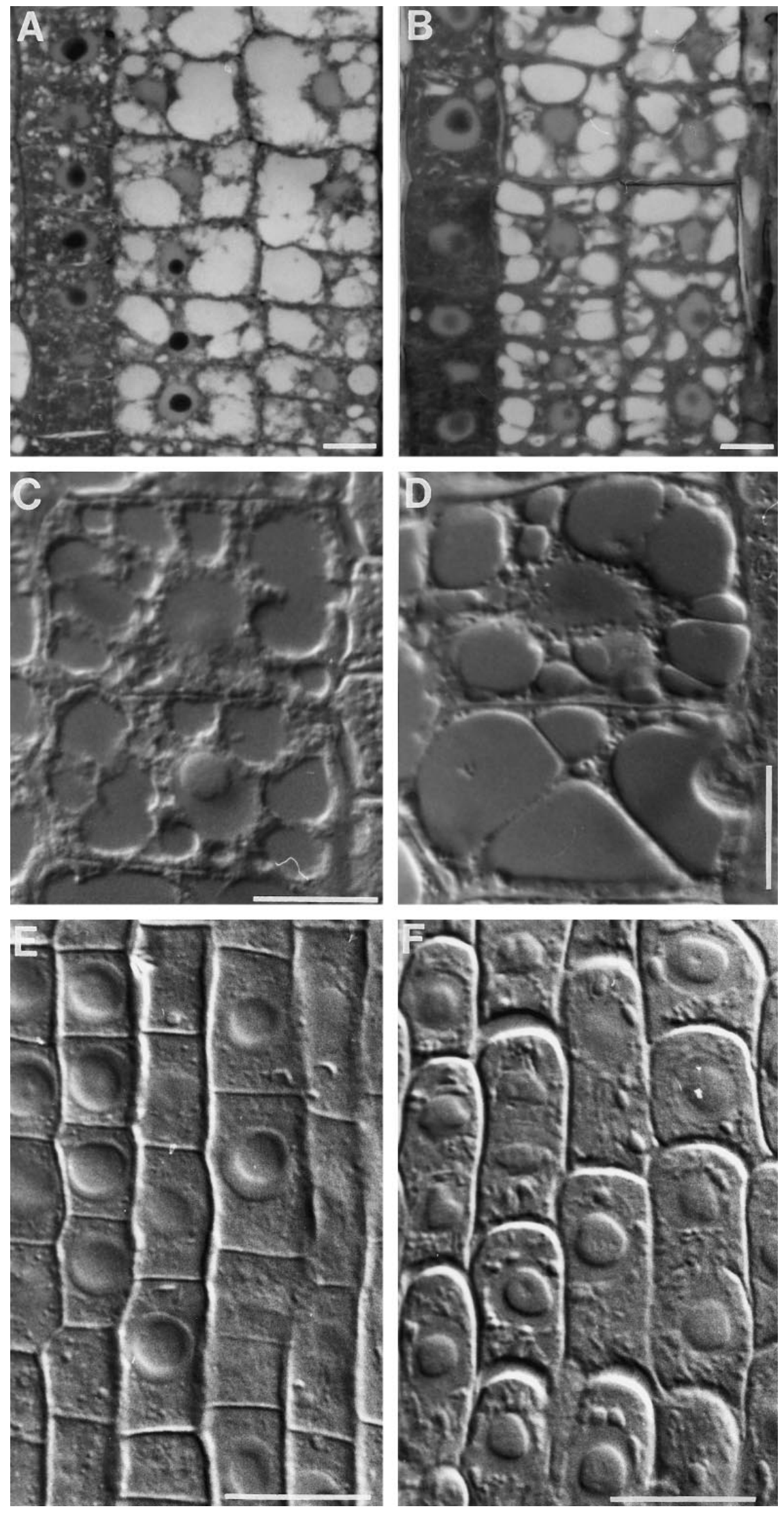

Fig. 5. Comparison of chemical fixation vs. plunge freezing on the appearance of cytoplasm in roots of $A$. thaliana. $(A, B)$ Sections, with embedment not removed, stained with toluidine blue of (A) chemically fixed and (B) cryofixed roots. Note the ragged appearance of the cytoplasm in chemically fixed roots. (C-F) Extracted sections viewed with Nomarski optics of $(C, E)$ chemically fixed and (D,F) plungefrozen roots. Note with cryofixation the fine trans-vacuolar strands in large cells and the appearance of many inclusions resembling membrane-bound organelles in dense mitotic cells. Nomarski images should be viewed so that the stands in (C) and (D) appear as ridges and the cell walls in (E) and (F) appear as valleys. Scale bars $=10 \mu \mathrm{m}, \quad(A, B) 765 \times, \quad(C, D) 1835 \times$, (E,F) $2140 \times$. 
meristems of five roots, that their average size per cell was not evidently related to the cell's distancefrom the epidermis, and that their size averaged $54 \pm 5.7 \mathrm{~nm}$ (mean + SD; $n=2310$ ). Although this average is only a rough estimate, nevertheless its magnitude is indicative of well-frozen material (Ryan, 1992). Evidently, the presence of ice crystals of this size in a specimen need not overwhelm images from light microscopy.

With arabidopsis roots prepared for light microscopy, we identified several criteria by which plunge freezing seems to surpass chemical fixation. The cryofixed cytoplasm appears in better condition than its chemically fixed counterpart, as judged from smooth cytoplasm and complex organelle shapes. A further difference between chemical and cryofixation concerns the need for DTT during methacrylate polymerization. Chemically fixed samples embedded in methacrylate require DTT to retain antigenicity, presumably because DTT prevents oxidative damage caused by methacrylate-free radicals created du ring polymerization (Baskin et al., 1992). However, cryofixed samples embedded in methacrylate retained antigenicity without DTT. This result suggests that sites in the sample subject to free radical attack are exposed or created by chemical fixation, through chemical reactions or structural rearrangements brought about by the fixative.

Furthermore, cryofixation led to a lifelike appearance of both actin and microtubule arrays. The cryofixed root cells, like pollen tubes and stamen hairs, had a fine actin reticulum that resembles the appearance of actin arrays in living plant cells (Hepler et al., 1993). Of course, local re-arrangements within this array due to ice crystallization may have occurred; nevertheless, compared with chemical fixation, where cortical and epidermal cells were totally unstained, cryofixation represents an improvement. Similarly, cryofixed cells had cortical microtubule arrays that resembled those in living cells, insofar as arrays with a given orientation were found that had numerous divergent microtubules (Fig. 3E). Arrays with such divergent microtubules have been rarely imaged with chemical fixation but have been reported to be frequent in living cells, injected with fluorescent tubulin (Hush et al., 1994; Yuan et al., 1994). Because the cytoskeleton is dynamic, with the half-life of microtubules in interphase plant cells being of the order of 1 min (Hush et al., 1994; Yuan et al., 1994), it is not surprising that chemical fixation, which takes many minutes to halt all cellular activity (Mersey \& McCully, 1978; He \& Wetzstein, 1995), fails to preserve cytoskeletal arrays completely.

Although cryofixed roots with poorly preserved areas occurred, these areas were obvious and could be disregarded. However, the major problem we encountered was the splitting apart of cell walls during sectioning. Substitution in tetrahydrofuran cau sed the same amount of splitting as in acetone; however, substitution in methanol or ethanol eliminated it completely. Nevertheless, in these solvents, cellular preservation was inferior compared with acetone. Results with ethanol could be improved by the inclusion of $4 \%$ paraformaldehyde, but remained less satisfactory than with acetone. Addition to substitution media of $1 \%$ DMP, a compound that removes water chemically (Kaeser, 1989), modestly improved the preservation of samples, but did not affect wall splitting. We believe that splitting in cryofixed roots is caused by fractures propagating through the straight cell walls. Trimming blocks with large faces minimized but did not eliminate wall splitting, probably by absorbing some energy from the microtome. Treatments such as methanol or ethanol substitution that eliminated cell wall splitting also cau sed walls to wrinkle slightly. W rinkles in the cell wall can be expected to slow or stop the propagation of fractures. Recently, we have found that sectioning with a diamond knife (histology grade) greatly reduced this splitting. Although the splitting is a nuisance, its occurrence does not invalidate observations made on intact regions or on groups of cells split away from their surroundings.

One possibly surprising finding, made for all of the material examined, is that inclusion of a fixative with acetone during freeze-substitution was not required. It is evident that samples are fixed through substitution in acetone, although the physical basis for this type of fixation is not completely understood. Inclusion of a fixative, usually osmium or glutaraldehyde, has been standard practice in freezesubstitution (Harvey, 1982; Hippe-Sanwald, 1993), but recently workers pursuing immunoelectron microscopy have demonstrated excellent ultrastructural preservation following freeze-substitution in pure solvents (Monaghan $\&$ Robertson, 1990; Lancelle \& Hepler, 1991; Nicolas \& Bassot, 1993). The reliability of fixation in pure acetone was of further concern for the methacrylate system used here, because sections are exposed to aqueous processing steps after the resin is dissolved. However, we found no loss of microtubule staining after disembedded sections of roots had been washed with high levels of detergent or salt, which indicates that the sections are reasonably stable. Nevertheless, further work is needed to establish to what extent they lose other constituents, such as soluble proteins, RNA or membranes.

We have demonstrated clear advantages of plunge-freezing over chemical fixation as a preparative technique for light microscopy for several plant cell types, including a multicellular organ. We have presented results for actin and tubulin, but we have also had successful results with other proteins. For example, in cryofixed pollen tubes embedded in methacrylate, three classes of myosin have been localized specifically, with important implications for mechanisms of organelle motility (Miller et al., 1995). Because the advantages we show relate generally to cellular morphology as well as to antigenicity, we expect that many proteins and other targets can be successfully localized with the described method. Likewise, we expect similar advantages exist for 
animal cells. Cryofixation of cultured animal cells always involves the difficulty of removing culture medium: too much remaining medium prevents optimal freezing of the cells, whereas too little leads to instant dehydration (Phillips \& Boyne, 1984). By inference from roots, we suggest that cryofixation and freeze substitution of animal cells for light microscopy will tolerate relatively large amounts of culture medium surrounding the cells at freezing. Application of plunge freezing to optical microscopy could becomea routine preparative technique, even for multicelluar specimens; we look forward to the ensuing series of discoveries.

\section{Acknowledgements}

We acknowledge Dale Callaham (University of Massachusetts) and Wayne Shoemaker (University of Missouri) for help in designing and building a cryofixation apparatus. We thank Ms Natalie Edwards (University of Missouri) for technical assistance, Dr Richard Cyr (Penn State) for the gift of the anti-actin monoclonal antibody, Dr Herman Meekes (University of Missouri) for help with electron microscopy, Dr Rick Howard (Dupont) for generously sharing his results prior to publication, Mr Tim Bourett (Dupont) for helpful discussions, and Professor Tom Phillips (University of Missouri) for reading the manuscript. This work was supported in part by the Cooperative States Research Service U.S. Department of A griculture under agreement nos. 9237304-7868 (with T.I.B.) and 94-37304-1180 (with P.K.H.), by a grant to T.I.B. from the U.S. Department of Energy (award no. 94ER20146), which does not constitute endorsement by that Department of views expressed herein, by NSF grant BBS-8719235 to the University of Massachusetts microscopy facility, and by NSF grant MCB-9304953 to P.K.H.

\section{References}

Andersland, J., Fisher, D., Wymer, C., Cyr, R. \& Parthasarathy, M. (1994) Characterization of a monoclonal antibody prepared against plant actin. Cell M otil. Cytoskel. 29, 339-344.

Angerer, L.M. \& Angerer, R.C. (1991) Localization of mRNAs by

in situ hybridization. Functional Organization of the Nudeus: a Laboratory Guide. Methods in Cell Biology, Vol. 35 (ed. by B. A. Hamalko \& S. C. R. Elgin), pp. 37-71. A cademic Press, San Diego.

Baskin, T.I. \& Bivens, N.J. (1995) Stimulation of radial expansion in arabidopsis roots by inhibitors of actomyosin and vesicle secretion but not by various inhibitors of metabolism. Planta, 197, 514521.

Baskin, T.I., Busby, C.H., Fowke, L.C., Sammut, M. \& Gubler, F. (1992) Improvements in immunostaining samples embedded in methacrylate: Localization of microtubules and other antigens throughout developing organs in plants of diverse taxa. Planta, 187, 405-413.

Baskin, T.I., Wilson, J.E., Cork, A. \& Williamson, R.E. (1994)
Morphology and microtubule organization in Arabidopsis roots exposed to oryzalin or taxol. Plant Cell Physiol. 35, 935-944.

Czymmek, K.J., Bourett, T.M. \& Howard, R.J. (1996) Immunolocalization of tubulin and actin in thick-sectioned fungal hyphae after freeze substitution fixation and methacrylate deembedment. J. Microsc. 181 (in press).

Dahl, R. \& Staehelin, L.A. (1989) High-pressure freezing for the preservation of biological structure: theory and practise. J. Electron M icrosc. Tech. 13, 165-174.

Ding, B., Turgeon, R. \& Parthasarathy, M.V. (1991) Routine cryofixation of plant tissue by propane jet freezing for freeze substitution. J. Electron M icrosc. Tech. 19, 107-117.

Feder, N. \& Sidman, R.L. (1958) Methods and principles of fixation by freeze substitution. J. Biophys. Biochem. Cytol. 4, 593-602.

Gilkey, J.C. \& Staehelin, L.A. (1986) A dvances in ultrarapid freezing for the preservation of cellular ultrastructure. J. Electron M icrosc. Tech. 3, 177-210.

Gubler, F. (1989) Immunofluorescence localization of microtubules in plant root tips embedded in butyl-methyl methacrylate. Cell Biol. Int. Rep. 13, 137-145.

Harvey, D.M.R. (1982) Freeze-substitution. J. M icrosc. 127, 209 221.

He, Y. \& Wetzstein, H.Y. (1995) Fixation induces differential tip morphology and immunolocalization of the cytoskeleton in pollen tubes. Physiol. Plant. 93, 757-763.

Hepler, P.K., Cleary, A.L., Gunning, B.E.S., Wadsworth, P., Wasteneys, G.O. \& Zhang, D.H. (1993): Cytoskeletal dynamics in living plant cells. Cell Biol. Int. Rep. 17, 127-142.

Hippe-Sanwald, S. (1993) Impact of freeze substitution on biological electron microscopy. M icrosc. Res. Tech. 24, 400-422.

Howard, R.J. \& O'Donnell, K.L. (1987) Freeze substitution of fungi for cytological analysis. Exp. M ycol. 11, 250-269.

Hush, J.M., Wadsworth, P., Callaham, D.A. \& Hepler, P.K. (1994) Quantification of microtubule dynamics in living plant cells using fluorescence redistribution after photobleaching. J. Cell Sci. 107, 775- 784.

Hyde, G.J., Lancelle, S., Hepler, P.K. \& Hardham, A.R. (1991) Sporangium structure in Phytophthora is disrupted after high pressure freezing. Protoplasma, 165, 203-208.

Kaeser, W. (1989) Freeze-substitution of plant tissues with a new medium containing dimethoxypropane. J. M icrosc. 154, 273- 278.

Kaeser, W., Koyro, H.-W. \& Moor, H. (1989) Cryofixation of plant tissues without pretreatment. J. M icrosc. 154, 279-288.

Kiss, J.Z. \& McDonald, K. (1993) Electron microscopy immunocytochemistry following cryofixation and freeze substitution. Antibodies in Cell Biology. M ethods in Cell Biology, Vol. 37 (ed. by D. J. A sai), pp. 311-341. A cademic Press, San Diego.

Lancelle, S.A., Callaham, D.A. \& Hepler, P.K. (1986) A method for rapid freeze fixation of plant cells. Protoplasma, 131, 153165.

Lancelle, S.A., Cresti, M. \& Hepler, P.K. (1987) Ultrastructure of the cytoskeleton in freeze-substituted pollen tubes of Nicotiana alata. Protoplasma, 140, 141-150.

Lancelle, S.A. \& Hepler, P.K. (1991) Association of actin with cortical microtubules revealed by immunogold localization in Nicotiana pollen tubes. Protoplasma, 165, 167-172.

Lancelle, S.A. \& Hepler, P.K. (1992) Ultrastructure of freezesubstituted pollen tubes of Lilium Iongiflorum. Protoplasma, 167, 215-230. 
McCully, M.E. \& Canny, M.J. (1985) The stabilization of labile configurations of plant cytoplasm by freeze-substitution. J. M icrosc. 139, 27-33.

Melan, M.A. \& Sluder, G. (1992): Redistribution and differential extraction of soluble proteins in permeabilized cultured cells. Implications for immunofluorescence microscopy. J. Cell Sci. 101, 731-743.

Menco, B.Ph.M. (1986) A survey of ultra-rapid cryofixation methods with particular emphasis on applications to freezefracturing, freeze-etching and freeze substitution. J. Electron Micros. Tech. 4, 177-240.

Mersey, B. \& McCully, M.E. (1978) Monitoring the course of fixation of plant cells. J. M icrosc. 114, 49-76.

Miller, D.D., Scordilis, S.P. \& Hepler, P.K. (1995) Identification and localization of three classes of myosins in pollen tubes of Lilium longiflorum and Nicotiana alata. J. Cell Sci. 108, 2549-2563.

Monaghan, P. \& Robertson, D. (1990) Freeze-substitution without aldehyde or osmium fixatives: ultrastructure and implications for immunocytochemistry. J. M icrosc. 153, 355-363.

Nicolas, M.-T. \& Bassot, J.-M. (1993) Freeze substitution after fastfreeze fixation in preparation for immunocytochemistry. M icrosc. Res. Tech. 24, 474-487.

Phillips, T.E. \& Boyne, A.F. (1984) Liquid nitrogen-based quick freezing: experiences with bounce-free delivery of cholinergic nerve terminals to a metal surface. J. Electron M icrosc. Tech. 1, 9-29.

Quintana, C. (1994) Cryofixation, cryosubstitution, cryoembedding for ultrastructural, immunocytochemical and microanalytical studies. M icron, 25, 63-99.

Raudaskoski, M., Rupeš, I. \& Timonen, S. (1991) Immunofluorescence microscopy of the cytoskeleton in filamentous fungi after quick-freezing and low temperature fixation. Exp. Mycol. 15, 167-173.
Ridge, R.W. (1990) A simple apparatus and technique for the rapidfreeze and freeze-substitution of single-cell algae. J. Electron Microsc. 39, 120-124.

Roberson, R.W. \& Vargas, M.M. (1994) The tubulin cytoskeleton and its sites of nucleation in hyphal tips of Allomyces macrogynus. Protoplasma, 182, 19- 31.

Ryan, K.P. (1992) Cryofixation of tissues for electron microscopy: A review of plunge freeze cooling methods. Scanning Microsc. 6, 715- 743.

Shopfer, C.R. \& Hepler, P.K. (1991) Distribution of membranes and the cytoskeleton during cell plate formation in pollen mother cells of Tradescantia. J. Cell Sci. 100, 717-728.

Sitte, H., Edelmann, L. \& Neumann, K. (1987) Cryofixation without pretreatment at ambient pressure. Cryotechniques in Biological Electron M icroscopy (ed. by R. A. Steinbrecht \& K. Zierold), pp. 87-111. Springer, Berlin.

Skaer, R.J. \& Whytock, S. (1976) The fixation of nuclei and chromosomes. J. Cell Sci. 20, 221-231.

Tiwari, S.C. \& Polito, V.S. (1988) Organization of the cytoskeleton in pollen tubes of Pyrus communis: a study employing conventional and freeze-substitution electron microscopy, immunofluorescence and rhodamine-phalloidin. Protoplasma, 147, 100112.

Van de Kant, H.J.G., Boon, M.E. \& De Rooij, D.G. (1988) Microwaveaided technique to detect bromodeoxyuridine in S-phase cells using immunogold-silver staining and plastic-embedded sections. Histochem. J. 20, 335-340.

Yuan, M., Shaw, P.J., Warn, R.M. \& Lloyd, C.W. (1994) Dynamic reorientation of cortical microtubules, from transverse to longitudinal, in living plant cells. Proc. Natl. Acad. Sci. USA, 91, 6050-6053. 